\title{
Kapton Polyimide-Based EEG Microelectrode Array and Interfaces for Mice Brainwave Recordings and Analysis
}

\author{
D. Gupta*a ${ }^{*}$ M. Islam ${ }^{\mathrm{a}}$, H. Nam ${ }^{\mathrm{b}}$, M. K. Lobo ${ }^{\mathrm{b}}$, F.-S. Choa ${ }^{\mathrm{a}}$ \\ ${ }^{a}$ Computer Science and Electrical Engineering, University of Maryland Baltimore County, 1000

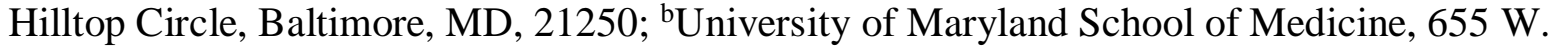 \\ Baltimore Street, Baltimore, MD, 21201
}

\begin{abstract}
There is a rise in the study of functional connectivity among various cortical regions and investigations to uncover causal links between a stimulus and the corresponding neural dynamics through electrophysiological imaging of the human brain. Animal model that exhibit simplistic representations of such networks open a doorway for such investigations and are gaining rapid popularity. In this study, we investigate and compare resting state network and auditory stimulus related activity with minimal invasive technology along computational spectral analysis on a C57/BL6 based mouse model. Somatosensory, motor and visual cortex are observed to be highly active and significantly correlated ( $p$-value<0.05). Moreover, given the spatial limitation due to small size of the mouse head, we also describe a low-cost and effective fabrication process for the mouse EEG Polyimide Based Microelectrodes (PBM) array. The easy-to-implement fabrication process involves transfer of the pattern on a copper layer of the Kapton film followed by gold electroplating and application of insulation paint. Acoustic stimulation is done by using tube extensions for avoiding electrical coupling to EEG signals. Unlike multi-electrode array type of invasive methods that are local to a cortical region, the methods established in this study can be used for examining functional connectivity analysis, neural dynamics and cortical response at a global level.
\end{abstract}

Keywords: Electroencephalography, Delta oscillations, PBM-array, Kapton, Fabrication, Correlation, Average Power, Polyimide

\section{INTRODUCTION}

Electroencephalography (EEG) is a useful noninvasive electrophysiological monitoring method for recording electrical activity of the brain. Unlike other brain-monitoring modalities, it has high temporal resolution that enables detailed study of the causal relationship between stimulation and the corresponding brain response. This also includes allowing the study of complex cortical networks interactions, such as the sensory, motor, visual, and cognitive functions of the cerebral cortex, at the milliseconds scale. These large-scale groups of neurons link together for normal functional behavior ${ }^{1}$. Hence, investigations with EEG can give useful insights towards a better understanding of the brain mechanisms, which can facilitate major breakthroughs for improved mental health too.

Although the first EEG recording dates back to $1924^{2}$ and there have been several studies since, however, a clear understanding of the brain mechanisms is still a mystery. The complexity lies in the fact that the human brain comprises of billions of neurons and connections as large as thousand times of that exists. Hence, investigating these questions with insect or animal models instead would be worthwhile given that its brain is a relatively smaller network size and that that mimics the human brain. For example, a growing body of literature is focusing on how somatosensory and motor cortical areas interact for better execution of normal behavior in insect and animal models ${ }^{3-5}$. However, these existing popular electrophysiological ${ }^{4}$ and optical ${ }^{5}$ methods for studying the mouse brain models, for example, are mostly invasive and are very specific to certain parts of the cortical areas. Hence, this makes it impossible to study a network function repetitively in the same animal or/and understand the overall large scale global brain networks. On the other hand, the minimally invasive EEG allows repetitive study of the overall global function network with high temporal resolution. 
In this study, we perform spectral analysis for all mouse EEG epochs during auditory stimulation (AS) and resting state (RS). We avoided averaging all EEG epochs for Event Related Potential (ERP) computation so as to study the brain dynamics. This is because although averaging may suppress the noise, it could also possibly make the actual signal weaker ${ }^{6}$. To the best of our knowledge, not much has been done on cortical network characteristics and response to an auditory stimulus in a mouse model with such minimally invasive EEG analysis. It is unclear how the spectral analysis changes with and without the auditory stimulus for various cortical regions. Here, we discuss specifically the delta wave activity response since it's associated with sleep and subconscious mind ${ }^{7,8}$ given that the mouse was anesthetized. We also describe our effective, simple, low-cost and easy-to-implement fabrication process for developing a basic polyimide based microelectrode array (PBM array) structure by using a Kapton film so as to overcome the spatial limitation given the small size of the mouse brain. This is followed by the EEG data recording and analysis.

\section{MATERIALS AND METHODS}

\subsection{Fabrication Process and Implementation}

We used a double-side copper-coated Kapton film was used as the substrate. Firstly, we laser printed our mask designed in AutoCAD on a transparent thin plastic sheet. Figure 1a shows this design pattern. We then transferred this pattern on one of the copper-side of Kapton film (see Figure 1b) by standard lithography followed by etching the exposed copper on both sides with $\mathrm{FeCO}_{3}$ and electroplating $\mathrm{Au}$ (gold) on the patterned copper layer. For lithography, we used AZ5214 image reversal photoresist (pre-baked at $90^{\circ} \mathrm{C}$, spin-coated at 4000 r.p.m., post-baked at $120^{\circ} \mathrm{C}, 1.4 \mu \mathrm{m}$ thick coat). The electrodes were $2 \mathrm{~mm}$ in diameter and wires were $1 \mathrm{~mm}$ wide. Finally, an insulating transparent paint was used to carefully cover the connecting wires but not the electrodes, leaving them exposed for signal retrieval during EEG recording from the mouse. The connections were done with a small socket to interface with the polyimide EEG cap interconnect. The 8-channel socket is soldered on another kapton film with wire expansion fabricated using the same fabrication process to spatially expand the 8 channels to larger contact pads with wider separation distance for easier wire soldering as shown in Figure 1c. These extensions were then connected to the KT88 equipment that has the Analog-to-Digital Converter (ADC), amplifier along the necessary software for digital EEG signal retrieval and data analysis.

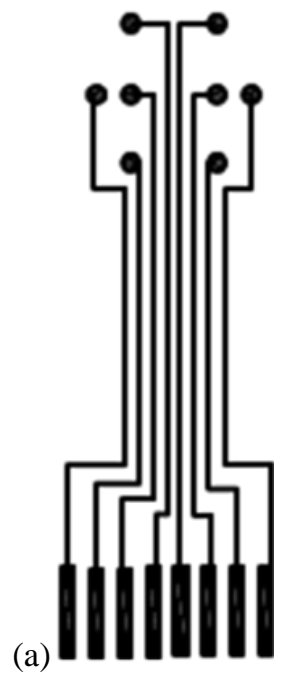

(b)

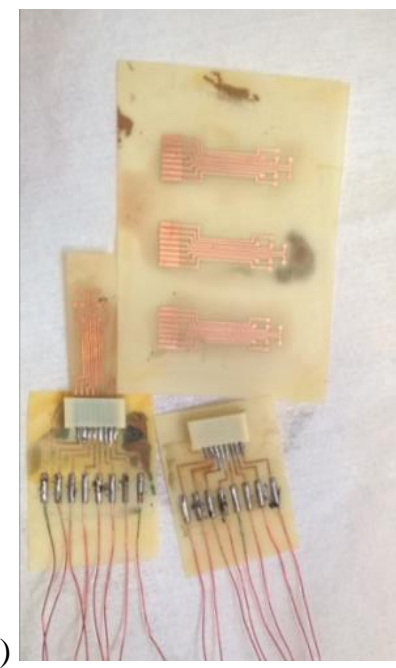

(c)

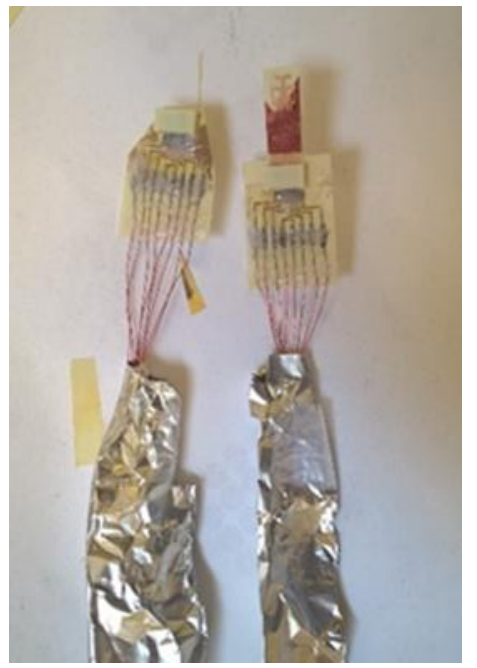

Figure 1 Our fast and easy-to-implement fabrication process results: (a) The mask design of electrodes with connecting wires. (b) Mask pattern transferred by using lithography on the Kapton film along the necessary extensions circuitry for connections with the EEG equipment (transparent paint for insulation is not visible). (c) Final completed product (right) with extension insulated by aluminum film (left and right). 


\subsection{Animal EEG Recording}

The C57/BL6J based male mice and aged 9-10 weeks were used in experiment following IACUC protocol \# 0517004 "Molecular and Functional Studies of Stress and Depression on the Brain" approved by UM Baltimore School of Medicine Office of Animal Welfare Assurance. We used isofluorane-based anesthesia to anesthetize our mouse. Positioning of the electrodes cannot be fixated on top of the mouse skin. Hence, we removed the top skin surgically and placed the electrodes on the skull of the mouse. Three $0.5 \mathrm{~mm}$ diameter screws were used to fixate the kapton film on top of the mouse skull. The debris from the skull was wiped off by saline-soaked cotton tips to enhance the adherence of the electrode to the skull of the mouse. The mouse head was then fixed by using a head-holder for avoiding movement causing noisy EEG with the EEG recording electrodes in place as shown in Figure 2. As mentioned in section 2.1, the EEG signal was digitally gathered by using the KT88 at a sampling rate of $100 \mathrm{~Hz}$.
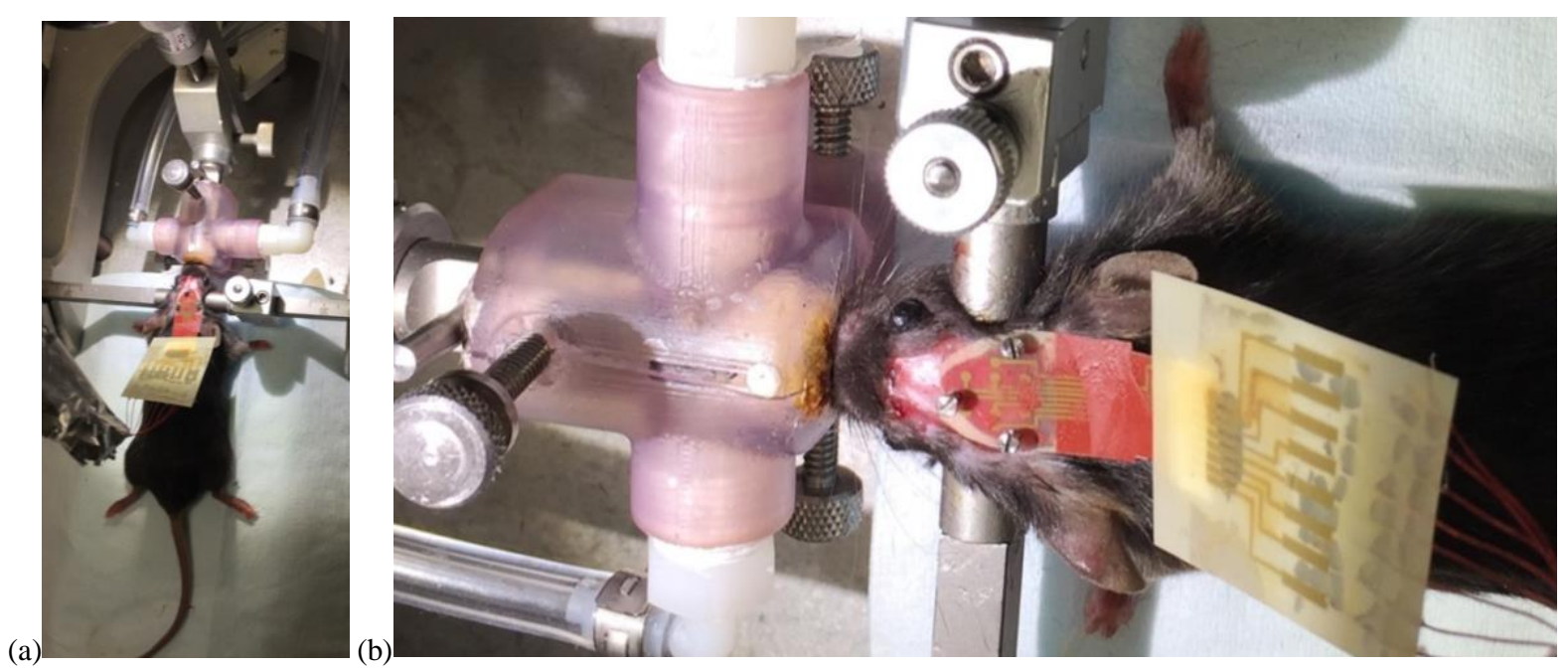

Figure 2. (a) Mouse fixed with head-holding clamping with EEG electrodes on Kapton film screwed to the top of the head after surgically skin-removal. (b) A zoomed-in view showing Kapton film based electrode array mounted on mouse's head.

\subsection{Acoustic Stimulation}

A repetitive tone of 5 seconds of duration with silent gaps of 5 seconds in between was generated using MATLAB. The tone was $1 \mathrm{KHz}$ modulated at $6 \mathrm{~Hz}$. For audio delivery to the mouse's ears, tube extensions were used to avoid electrical coupling into the EEG data. One ear was stimulated at a time.

\subsection{EEG Data Analysis}

The Kapton PBM array was mounted such that, other than two reference electrode channels, EEG electrode was mounted on top of the Right Visual Cortex (RVC), Left Motor Cortex (LMC), Right Somatosensory Cortex (RSC), Left Somatosensory Cortex (LSC) and Left Visual Cortex (LVC) during the auditory stimulus (AS) and the resting state (RS) as also shown in Figure 3a. The electrode channel mounted on the Right Motor Cortex (RMC) was contaminated and was hence, eliminated from the study. We focused on the Delta wave activity specifically because it is associated with the resting or sleeping state ${ }^{7,8}$ considering that the mouse was anesthetized. Average power computation for all EEG epochs for the $1 \mathrm{~Hz}$ to $4 \mathrm{~Hz}$ delta wave band was computed for both audio stimulus (AS) and resting state (RS). We did not average EEG epochs for computing Event Related Potential (ERP) because we wanted to consider the brain dynamics by observing every instance because with ERP, averaging over several epochs although suppresses noise but it also could possibly bury the signal underneath and make the actual signal weaker ${ }^{6}$. Hence, we computed average power for all 10 EEG epochs of AS and 10 EEG epochs of RS. Finally, we performed 2-sample t-test for the average power of the two states (AS and RS) to identify those EEG channels whose delta average power values significantly differed between the two states (AS and 
RS). We also computed correlation and related p-values among the 5 channels to evaluate if any channels were significantly correlated. All computations were done using MATLAB and EEGLAB ${ }^{9}$.

\section{RESULTS AND DISCUSSION}

On performing 2-sample t-test, we found that average power of neural delta wave activity was significantly suppressed during the Auditory Stimulus (AS) as compared with the Resting State (RS) in the LMC (p-value $=0.0291<0.05$ ) and LSC $(p$-value $=0.0281<0.05)$ areas. We know that the delta oscillations are correlated with the resting-state network ${ }^{7,8}$. Hence, this clearly indicates that the auditory stimulus (AS) significantly activated the LMC and the LSC and suppressed the resting-state network. Figure 4 shows boxplot for average power values associated with delta neural oscillations of the 5 channels during AS and RS. Clearly, both left and right visual cortex have relatively the highest variance and average power values as compared with other cortical regions for both AS and RS related EEG epochs. Furthermore, across all state (AS and RS) epochs (p-value $<0.05$ for all epochs), RVC was highly correlated with the RSC with an average correlation value of 0.87 and the LVC was highly correlated with the LSC with an average correlation value of 0.71 . This implies that the visual cortex in both hemispheres has high delta oscillations related power values and variance as compared with other cortical regions during both AS and RS as we can see in Figure 4, and that somatosensory and the visual cortex are highly correlated in an ipsilateral manner (p-value $<0.05$ during all AS and RS segments i.e. epochs) with the above specified average correlation values. We believe that this is due to the fact that the mouse's eyes were wide open throughout the experiment (see Figure 2). Lastly, LMC and LSC are strongly correlated with average correlation value of 0.77 and pvalue $<0.05$ for all AS and RS epochs. We believe that this is associated with the fluctuation between the auditory stimulus input and the resting state. A summary of these correlations is shown in Figure $3 \mathrm{~b}$.

(a)

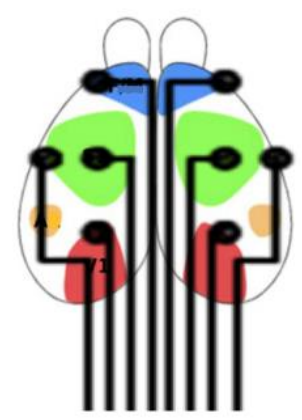

(b)

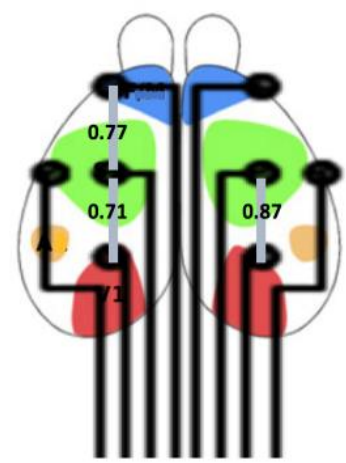

Figure 3. (a) Schematic view of EEG PBM electrode placement on the mouse cortex. Blue: Motor Cortex, Green: Somatosensory Cortex, Red: Visual Cortex. At the edge of the somatosensory cortex are the reference electrodes. (b) Highly correlated channel areas marked with respective correlation values 0.77 (LMC and LSC), 0.71 (LVC and LSC) and 0.87 (RVC and RSC). 


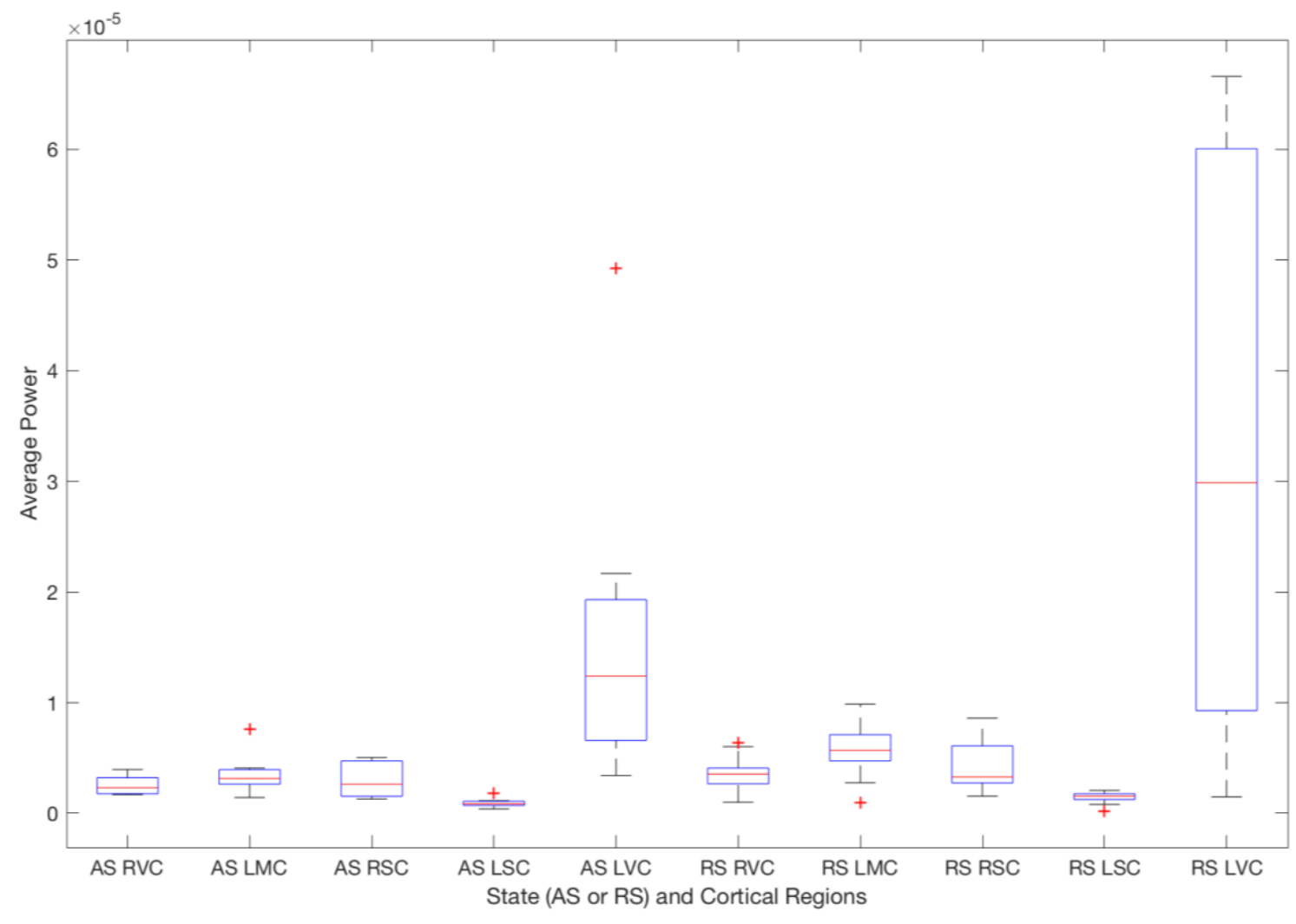

Figure 4. Box plot for average power values associated with Delta Oscillations during Auditory Stimulus (left 5 channels) and Resting State (right 5 channels) Abbreviations: AS = Auditory Stimulus, RVC $=$ Right Visual Cortex, LMC $=$ Left Motor Cortex, RSC = Right Somatosensory Cortex, LSC = Left Somatosensory Cortex, LVC = Left Visual Cortex, RS = Resting State.

\section{CONCLUSION AND FUTURE WORK}

We demonstrated simple, easy, low-cost and efficient method for fabricating mouse EEG PBM array. We successfully used it for recording EEG data recording. We computationally analyzed the EEG data and found that average power related to the delta oscillations is suppressed significantly in the LMC and LSC areas during the AS (p-value $<0.05$ ) as compared with the resting state network and that they are most highly correlated (0.87) as compared with all other correlated regions. Furthermore, we saw that the visual cortex and somatosensory areas are highly correlated (ipsilateral) and that delta average power is the highest in visual cortex as the mouse's eyes were wide open during the experiment. We avoided ERP computation and did dynamic brain EEG analysis for every epoch. To the best of our knowledge, this is the first study to investigate and provide functionally connectivity of a mouse brain globally via minimally invasive technology. For future work, we intend to gather more EEG data epochs and study other wave patterns and characteristics.

\section{REFERENCES}

[1] Bressler, S. L. and Tognoli, E., "Operational principles of neurocognitive networks," International Journal of Psychophysiology 60(2), 139-148 (2006). 
[2] Haas, L. F., "Hans berger (1873-1941), richard caton (1842-1926), and electroencephalography," Journal of Neurology, Neurosurgery \& Psychiatry 74(1), 9-9 (2003).

[3] Turner-Evans, D., Wegener, S., Rouault, H., Franconville, R., Wolff, T., Seelig, J. D., Druckmann, S. and Jayaraman, V., “Angular velocity integration in a fly heading circuit,” Elife 6 (2017).

[4] Benison, A. M., Rector, D. M. and Barth, D. S., "Hemispheric mapping of secondary somatosensory cortex in the rat," Journal of neurophysiology 97(1), 200-207 (2007).

[5] Ferezou, I., Bolea, S. and Petersen, C. C., "Visualizing the cortical representation of whisker touch: voltage-sensitive dye imaging in freely moving mice," Neuron 50(4), 617-629 (2006).

[6] Meng, Q., Gupta, D., Wudenhe, A., Du, X., Hong, L., Choa, F. S., "Three-Dimensional EEG Signal Tracking for Reproducible Monitoring of Self-Contemplating Imagination," Advances in Science Technology and Engineering Systems Journal, vol. 2, no. 3, pp. 1634-1646 (2017).

[7] Vyazovskiy, V. V., Olcese, U., Hanlon, E. C., Nir, Y., Cirelli, C. and Tononi, G., "Local sleep in awake rats," Nature 472(7344), 443-447 (2011).

[8] Hiltunen, T., Kantola, J., Elseoud, A. A., Lepola, P., Suominen, K., Starck, T., Nikkinen, J., Remes, J., Tervonen, O. and Palva, S., "Infra-slow EEG fluctuations are correlated with resting-state network dynamics in fMRI," Journal of Neuroscience 34(2), 356-362 (2014).

[9] Delorme, A. and Makeig, S., "EEGLAB: an open source toolbox for analysis of single-trial EEG dynamics including independent component analysis," Journal of Neuroscience Methods 134(1), 9-21 (2004). 